\title{
ABSTRACT
}

\section{A STUDY OF SEMANTIC RELATION BY THE ASSOCIATION METHOD AND THE SEMANTIC DIFFERENTIAL METHOD.}

\author{
-Analysis of Synonym and Antonym-
By Morimoto, Hiroshi, Kashu, Kan and Nakata, Yoshiro
Kobe Yamate Women's
Junior College
Kansei Gakuin University

This study is a part of the series of study on semantic relation.

Problem. In this first report, an attempt is made to analyse objectively the difference of synonym and antonym by the association method and the Semantic Differential method proposed by Osgood and Suci.

Method. 10 words, in which some synonyms and antonyms were involved, were selected from "Kashu' and Kubo's list of association-value of threesyllable verbs". We adopted the Semantic Differential method to analyse these words, but Osgood and Suci's method is somewhat modified by us. The subjects were 20 students.

Results. Besides association-value, difference scores and factor loadings (measured by the Semantic Differential) were used as the measures of semantic relation.
The results are as follows :

(1) In synonym, association-value was high, difference scores were low, and common factor was extracted.

(2) In antonym, though association-value was high as well as in the case of synonym, difference scores were high, and the results of factor analysis showed the opposite orientation.

Discussion and Conclusion. From the above findings, it is concluded that antonym was obviously distincted from synonym, and that Semantic Differential was especially useful to analyse the opposition quantitatively as Osgood referred to. Further, the possibilities of the application of the Semantic Differential to the study of the various types of the opposition were recognized.

\section{THE TYPOLOGICAL ANALYSIS OF THE SOCIAL ATTITUDES}

\author{
By Tanaka, Kunio and Matsuyama, Yasuo \\ Kobe City Univ. Osaka Univ. of Liberal \\ of Foreign Studies Arts and Education
}

\section{Problem}

The purpose of this experiment was to throw light upon the type-factor of the social attitudes, using the inverted technique of the factor-analysis. In this experiment, we put emphasis on the following points. The first point was to find out the attitude- 